\title{
Role of H3K27 methylation in the regulation of IncRNA expression
}

\author{
Susan C Wu ${ }^{1,2}$, Eric M Kallin ${ }^{1,2,3}$, Yi Zhang ${ }^{1,2}$ \\ ${ }^{1}$ Howard Hughes Medical Institute, ${ }^{2}$ Department of Biochemistry and Biophysics, Lineberger Comprehensive Cancer Center, Uni- \\ versity of North Carolina at Chapel Hill, Chapel Hill, NC 27599-7295, USA
}

Once thought to be transcriptional noise, large non-coding RNAs (IncRNAs) have recently been demonstrated to be functional molecules. The cell-type-specific expression patterns of IncRNAs suggest that their transcription may be regulated epigenetically. Using a custom-designed microarray, here we examine the expression profile of IncRNAs in embryonic stem (ES) cells, lineage-restricted neuronal progenitor cells, and terminally differentiated fibroblasts. In addition, we also analyze the relationship between their expression and their promoter H3K4 and H3K27 methylation patterns. We find that numerous IncRNAs in these cell types undergo changes in the levels of expression and promoter H3K4me3 and H3K27me3. Interestingly, IncRNAs that are expressed at lower levels in ES cells exhibit higher levels of H3K27me3 at their promoters. Consistent with this result, knockdown of the H3K27me3 methyltransferase Ezh2 results in derepression of these IncRNAs in ES cells. Thus, our results establish a role for Ezh2-mediated H3K27 methylation in IncRNA silencing in ES cells and reveal that IncRNAs are subject to epigenetic regulation in a similar manner to that of the protein-coding genes.

Keywords: IncRNA; histone methylation; Polycomb repressive complex 2

Cell Research (2010) 20:1109-1116. doi:10.1038/cr.2010.114; published online 3 August 2010

\section{Introduction}

Recent studies have revealed that the mammalian 'transcriptome' is much more substantial and complex than was originally believed. The ENCODE project estimates that over $90 \%$ of all nucleotides within the human genome can be transcribed [1]. Although transcription appears to be quite pervasive, only $15 \%$ of the genome experiences stable transcription in human cell lines [2]. Nevertheless, protein-coding genes account for just $1-2 \%$ of the genome [3], suggesting that cells dedicate a considerable amount of energy to produce transcriptional 'dark matter' [4].

Although small RNAs (microRNAs, PIWI-associated RNAs, and small interfering RNAs) and housekeeping RNAs (transfer RNAs and ribosomal RNAs) account for

Correspondence: Yi Zhang

Tel: +1-919-843-8225; Fax: +1-919-966-4330

E-mail: yi_zhang@med.unc.edu

${ }^{3}$ Current address: Center for Genomic Regulation, 08003 Barcelona, Spain Received 14 May 2010; revised 7 June 2010; accepted 17 June 2010;

published online 3 August 2010 some non-protein coding transcripts, the vast majority are large non-coding RNAs (lncRNAs) that are greater than 200 nucleotides in length [5]. Although lncRNAs were once considered to be transcriptional noise, several features suggest that lncRNAs may play important roles in various processes. These features include (1) cell-typespecific expression patterns [6-8]; (2) distinct subcellular localizations [7]; (3) link to various diseases [9]; and (4) evolutionary selection of the lncRNA sequence $[10,11]$. Indeed, recent studies have uncovered functional roles for lncRNAs in diverse biological processes including $\mathrm{X}$-inactivation, imprinting, Hox gene regulation, and the development of certain diseases [9, 12-15].

Epigenetic modifications, including DNA methylation, histone modifications, and chromatin remodeling, are believed to contribute to the cell-type-specific expression patterns of protein-coding genes $[16,17]$. Interestingly, a previous study has identified many genomic regions that contain the active histone modification marks H3K4me3H3K36me3, which could not be attributed to proteincoding genes [10]. While this suggests that lncRNAs may be regulated epigenetically in a similar way to that of protein-coding genes, this has not been tested directly. 
To begin to understand the mechanism by which lncRNAs exhibit cell-type-specific expression, we compared the expression profiles of lncRNAs in mouse embryonic stem (ES) cells, neuronal progenitors (NPCs), and terminally differentiated fibroblasts and analyzed the relationship between their expression and their promoter $\mathrm{H} 3 \mathrm{~K} 4 \mathrm{me} 3$ and H3K27me3 levels. We found that these histone modifications undergo dynamic changes in a celltype-specific manner. In addition, we found that Ezh2mediated $\mathrm{H} 3 \mathrm{~K} 27$ methylation plays an important role in

A

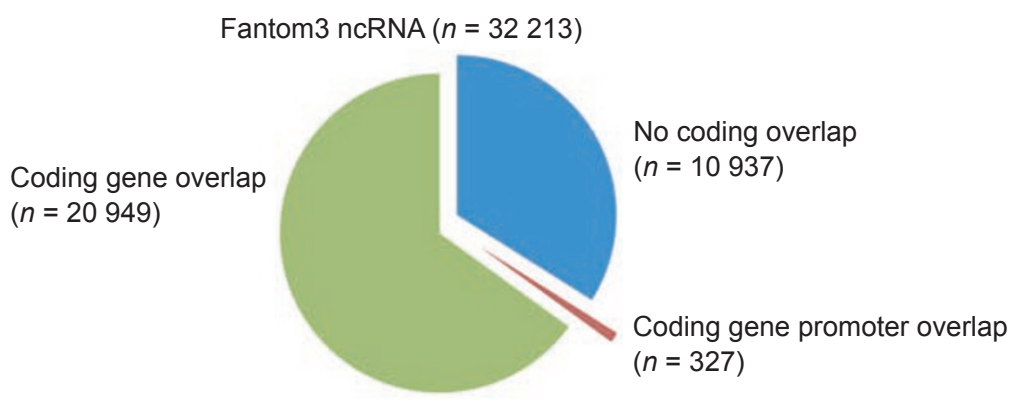

B

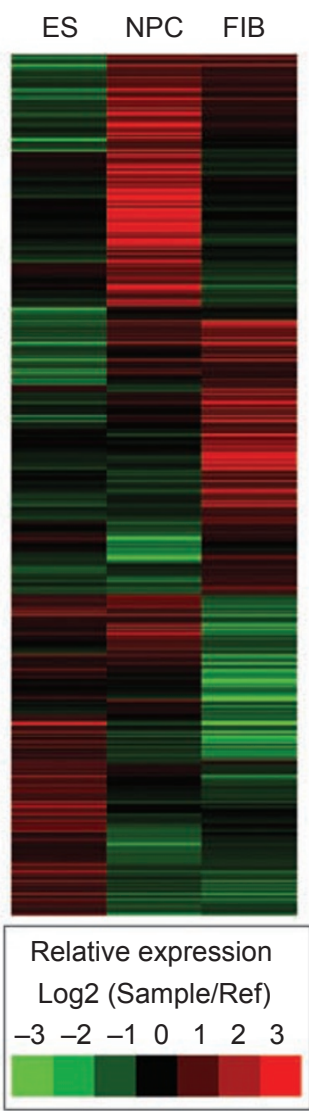

C

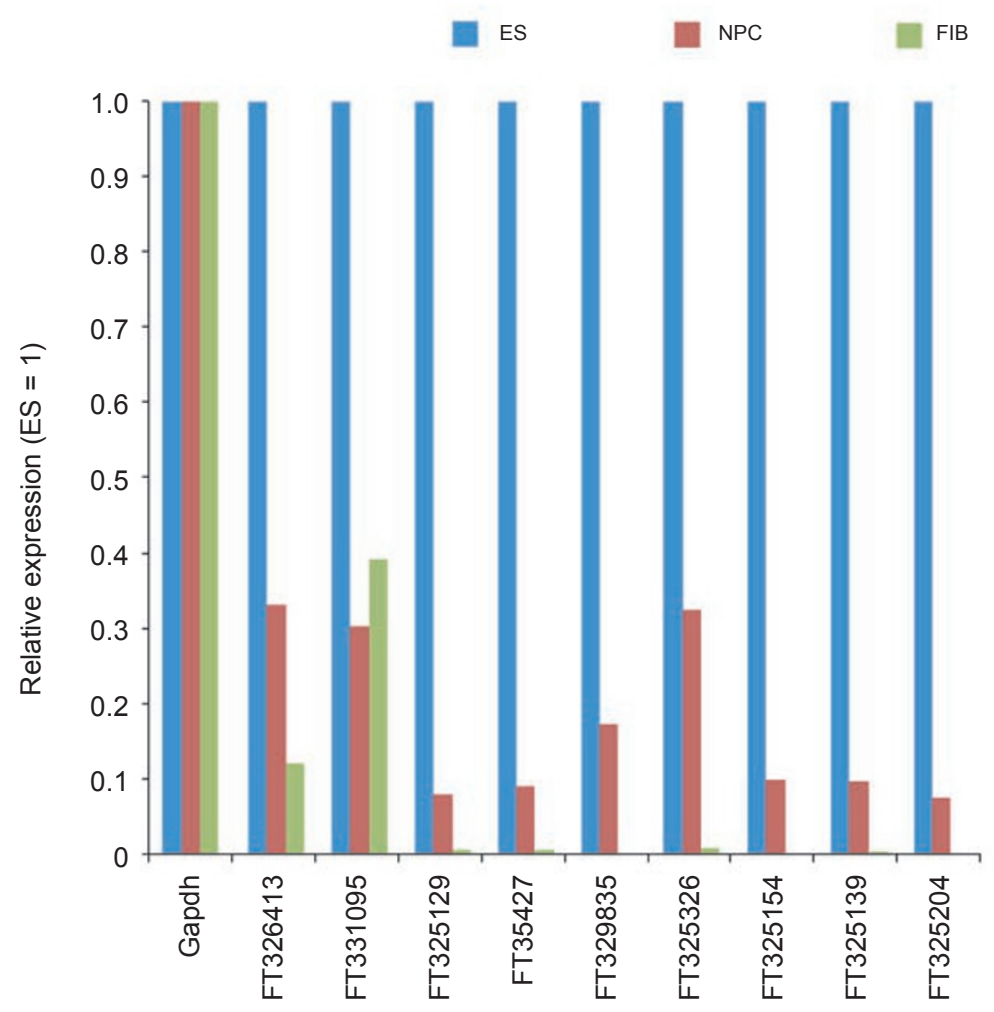

Figure 1 IncRNAs are differentially expressed in ES cells, NPCs, and fibroblasts. (A) Pie chart representation of the distribution of IncRNAs in the mouse genome. Independent Fantom3 IncRNA entries were scanned for overlap with promoters, defined as -1 to $+1 \mathrm{~kb}$ surrounding the TSS, and coding regions of annotated genes from the REFSEQ database. (B) Heat map presentation of the expression profile of IncRNAs in ES cells, NPCs, and fibroblasts. The data presented for each cell type were compared to universal reference RNA and were subjected to uncentered hierarchical clustering. ES: embryonic stem cells, NPC: neuroprogenitors, FIB: fibroblasts. (C) RT-qPCR validation of microarray data for randomly chosen IncRNAs that exhibited at least two-fold elevated transcription in ES versus both NPCs and fibroblasts. 
silencing lncRNAs in ES cells. Our results suggest that lncRNAs are epigenetically regulated in a similar manner to that of protein-coding genes.

\section{Results}

Expression profiling of lncRNAs in ES cells, NPCs, and fibroblasts

Of the 34030 lncRNAs annotated in the Fantom3 database, 32213 (95\%) were mapped to the UCSC mm8 assembly of the mouse genome. To determine whether lncRNAs are differentially expressed in cells at different differentiation stages, we analyzed their expression in mouse ES cells, lineage-restricted neuroprogenitors (NPCs), and terminally differentiated fibroblasts using a custom-designed microarray containing oligonucleotide probes targeting these lncRNAs (Supplementary information, Table S1). Given that multiple transcriptional units can coexist within the same genomic space [18-22], lncRNAs may be regulated indirectly by their location within the same transcriptional unit of a protein-coding gene. To simplify the analysis, we focused on the subset of lncRNAs that did not overlap with the promoter (defined as $-1 \mathrm{~kb}$ to $+1 \mathrm{~kb}$ surrounding the transcription start site (TSS)) or the coding region of annotated genes. We found that the majority $(65 \%, n=20949)$ of mapped
lncRNAs annotated in the Fantom3 database overlapped with coding regions of genes annotated in the RefSeq database. A small fraction $(1 \%, n=327)$ overlapped with gene promoters. The remaining lncRNAs $(34 \%, n=10$ 937) showed no overlap with annotated promoters or coding regions (Figure 1A). These lncRNAs were used for all subsequent analyses.

We found that 3270 (10.2\%) unique lncRNAs exhibit at least a two-fold change when compared from one cell type to another (Figure 1B). IncRNAs that exhibited at least a two-fold change in ES cells compared to both NPCs and fibroblasts were randomly selected and confirmed by quantitative PCR analysis (Figure 1C). These studies indicate that at least a subset of lncRNAs exhibit cell-type-specific expression.

H3K4me3 and H3K27me3 distribution in lncRNA promoters in ES, NPC, and fibroblasts

Given that epigenetic mechanisms contribute to celltype-specific expression of protein-coding genes, we asked whether lncRNAs are epigenetically regulated. The Trithorax and Polycomb group of proteins are key developmental players responsible for lineage-specific gene expression [23]. Some of these proteins participate in multi-protein complexes that are responsible for the trimethylation of lysine 4 and 27 of histone H3 (H3K4me3
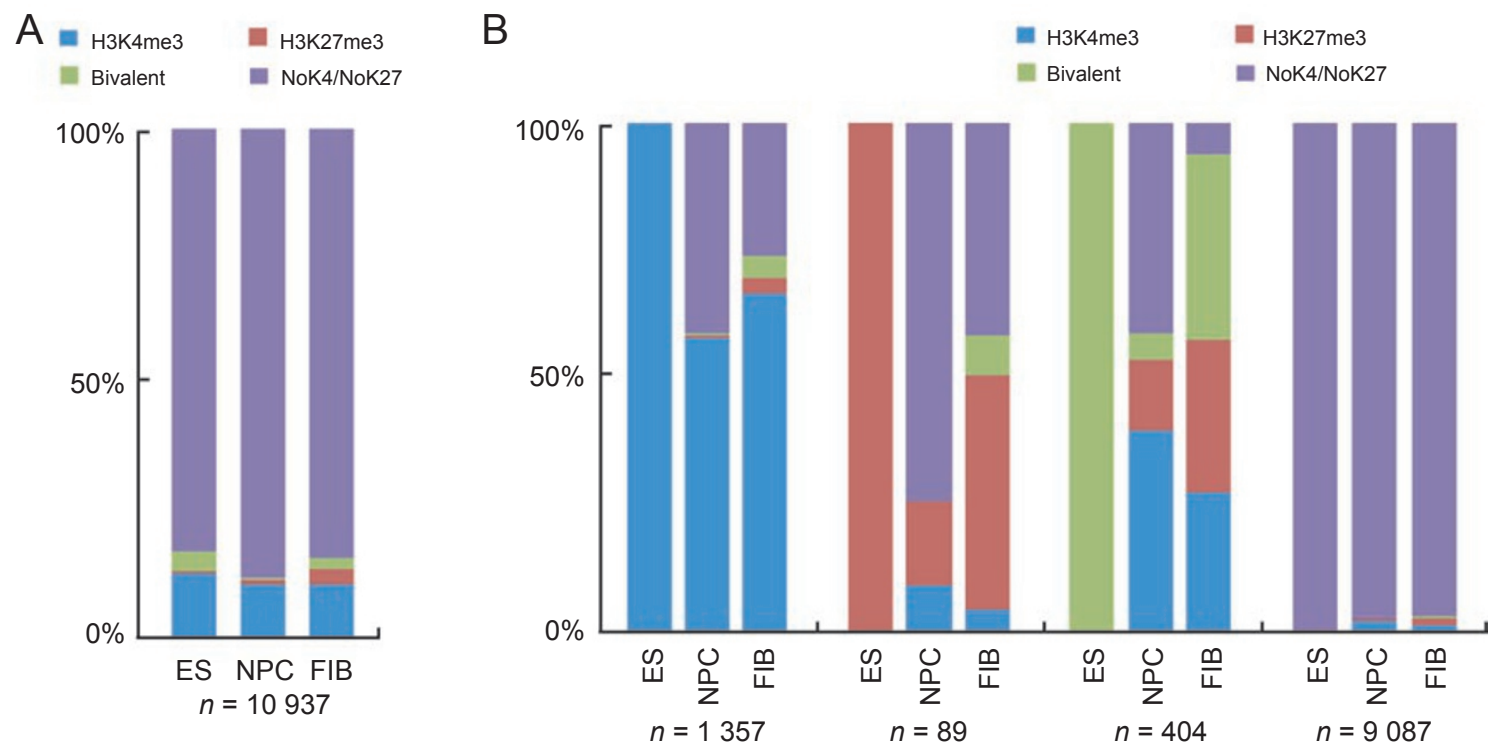

Figure 2 IncRNA promoter H3K4me3 and H3K27me3 patterns in ES cells, NPCs, and fibroblasts. Distribution of H3K4me3 and H3K27me3 at IncRNA promoters in ES cells, NPCs, and fibroblast cells for (A) all IncRNAs examined and (B) IncRNAs grouped by their epigenetic modification state in ES cells and then assessed for modification in both NPC and fibroblast cells. The presence or absence of $\mathrm{H} 3 \mathrm{~K} 4 \mathrm{me} 3$ and $\mathrm{H} 3 \mathrm{~K} 27 \mathrm{me} 3$ peaks was assessed within the promoters of IncRNAs that did not overlap with annotated REFSEQ genes $(n=10$ 937) in mouse ES cells, NPCs, and fibroblasts. H3K4me3 only, blue; H3K27me3 only, red; H3K4me3 and H3K27me3 (bivalent), green; no H3K4me3 and no H3K27me3, purple. 
and $\mathrm{H} 3 \mathrm{~K} 27 \mathrm{me} 3$ ), with $\mathrm{H} 3 \mathrm{~K} 4 \mathrm{me} 3$ and $\mathrm{H} 3 \mathrm{~K} 27 \mathrm{me} 3$ correlating with transcriptional activation and repression, respectively [17]. Specific genomic regions in ES cells are marked with both $\mathrm{H} 3 \mathrm{~K} 4 \mathrm{me} 3$ and $\mathrm{H} 3 \mathrm{~K} 27 \mathrm{me} 3$ and are termed 'bivalent' domains, which are transcriptionally inactive regions that are poised for transcriptional activation [24].

Taking advantage of previously published genomewide location data for $\mathrm{H} 3 \mathrm{~K} 4 \mathrm{me} 3$ and $\mathrm{H} 3 \mathrm{~K} 27 \mathrm{me} 3$ [25], we examined these two histone methylation marks at the promoters of lncRNAs in mouse ES cells, NPCs, and fibroblasts (Figure 2A). We found a small group of unique lncRNAs $(17 \%)$ that contain these modifications. In ES cells, roughly $80 \%$ of all $\mathrm{H} 3 \mathrm{~K} 27 \mathrm{me} 3$-positive lncRNA promoters also contain $\mathrm{H} 3 \mathrm{~K} 4 \mathrm{me} 3$, which is similar to previous reports on protein-coding genes [24]. In contrast to H3K27me3, less than a quarter of all H3K4me3positive promoters are bivalent, suggesting that most H3K4me3-positive lncRNAs may have housekeeping functions or are important for ES cell identity. Overall, ES cells contained more bivalent lncRNA promoters when compared to NPCs and fibroblasts. In addition, $\mathrm{H} 3 \mathrm{~K} 27 \mathrm{me} 3$ alone is notably enriched in fibroblasts compared to ES cells and NPCs, indicating that this subset of lncRNAs may have to be silenced in terminally differentiated cells.

We then asked whether lncRNAs containing these modifications retain the same modification or if they undergo epigenetic changes when ES cells become lineagerestricted (NPC) or terminally differentiated (fibroblasts). Unlike those lacking modifications, many lncRNAs that possess H3K4me3, H3K27me3, or both do undergo dynamic changes (Figure 2B). Interestingly, while more than half of the lncRNAs possessing H3K4me3 in ES cells retain this mark, the majority of the bivalent lncRNAs in ES cells commit to either the active or the repressive mark in NPCs and fibroblasts. This suggests that the lncRNAs retaining $\mathrm{H} 3 \mathrm{~K} 4 \mathrm{me} 3$ may have housekeeping functions, while those with the bivalent mark in ES cells, but committing to the active or repressive mark in NPCs or fibroblasts, may have important roles in cell lineage commitment. On the other hand, the group of lncRNAs that lose the H3K4me3 mark in NPCs and fibroblasts may be interesting for future studies, as these may be important for ES cell identity. It is interesting to note that most lncRNAs containing only H3K27me3 in ES cells appear to lose this modification in NPCs and fibroblasts, particularly in NPCs (Figure 2B).

Promoter H3K4me3 and H3K27me3 correlate with IncRNA transcription levels

We next set out to examine whether changes in $\mathrm{H} 3 \mathrm{~K} 4$

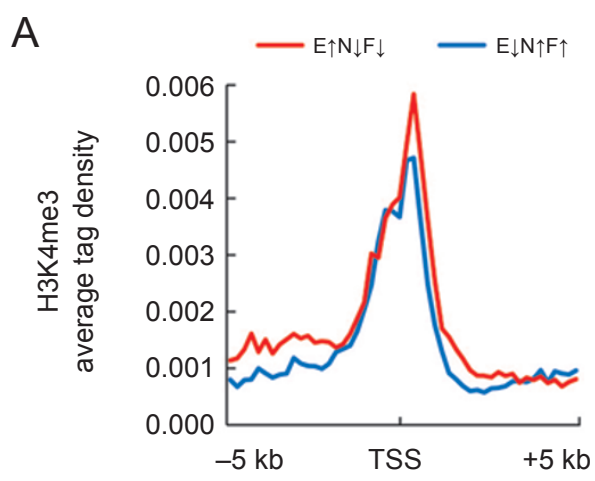

B

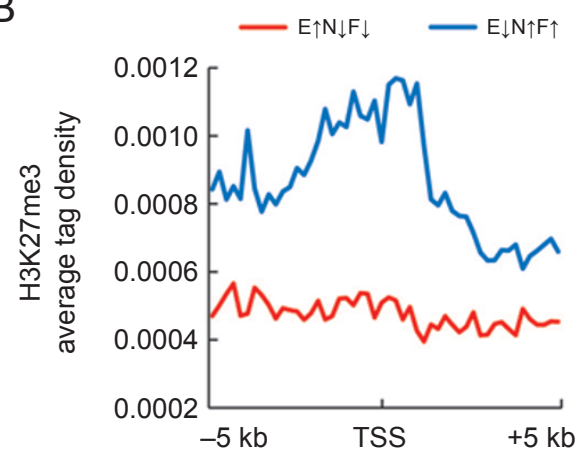

C

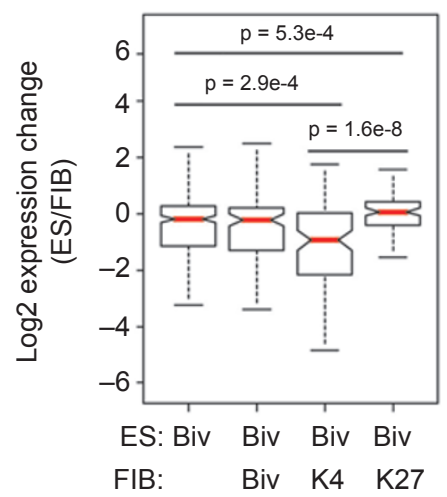

Figure 3 Changes in promoter H3K4me3 and H3K27me3 levels are correlated with IncRNA expression. The average H3K4me3 (A) and H3K27me3 (B) tag density across the promoters of IncRNAs in ES cells grouped by relative expression in ES versus NPC and fibroblast. The $X$-axis represents the region spanning -5 to $+5 \mathrm{~kb}$ away from the IncRNA transcription start site (TSS). The red line indicates IncRNAs that exhibit at least two-fold higher expression in ES cells relative to both NPCs and fibroblast cells, $\mathrm{E} \uparrow \mathrm{N} \downarrow \mathrm{F} \downarrow(n=1511)$. The blue line indicates IncRNAs that exhibit at least two-fold lower expression in ES cells relative to both NPCs and fibroblast cells, $\mathrm{E} \downarrow \mathrm{N} \uparrow \mathrm{F} \uparrow(n=1$ 696). (C) Box plot presentation of expression change (ES/FIB) in bivalentmarked IncRNAs in ES cells $(n=321)$, which are further divided into three groups according to their promoter H3K4 and H3K27 methylation state in fibroblast cells: H3K4me3 and H3K27me3, Biv $(n=115)$; H3K4me3 only, K4 $(n=98) ;$ H3K27me3 only, K27 ( $n$ $=89$ ). Significant $P$ values as determined by Wilcoxon signedrank test are indicated. 
A

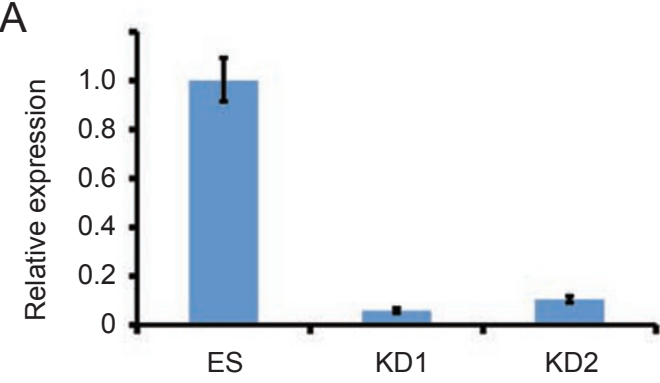

B

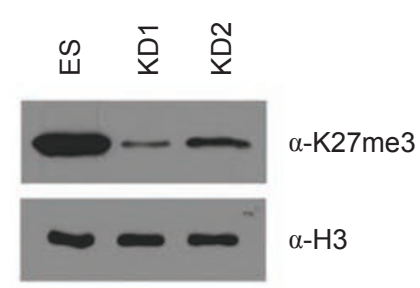

C

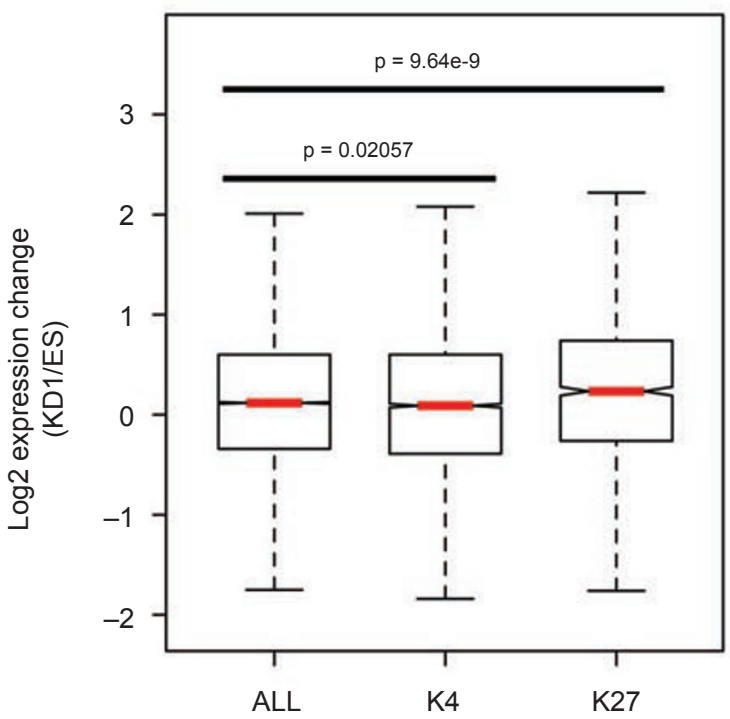

D

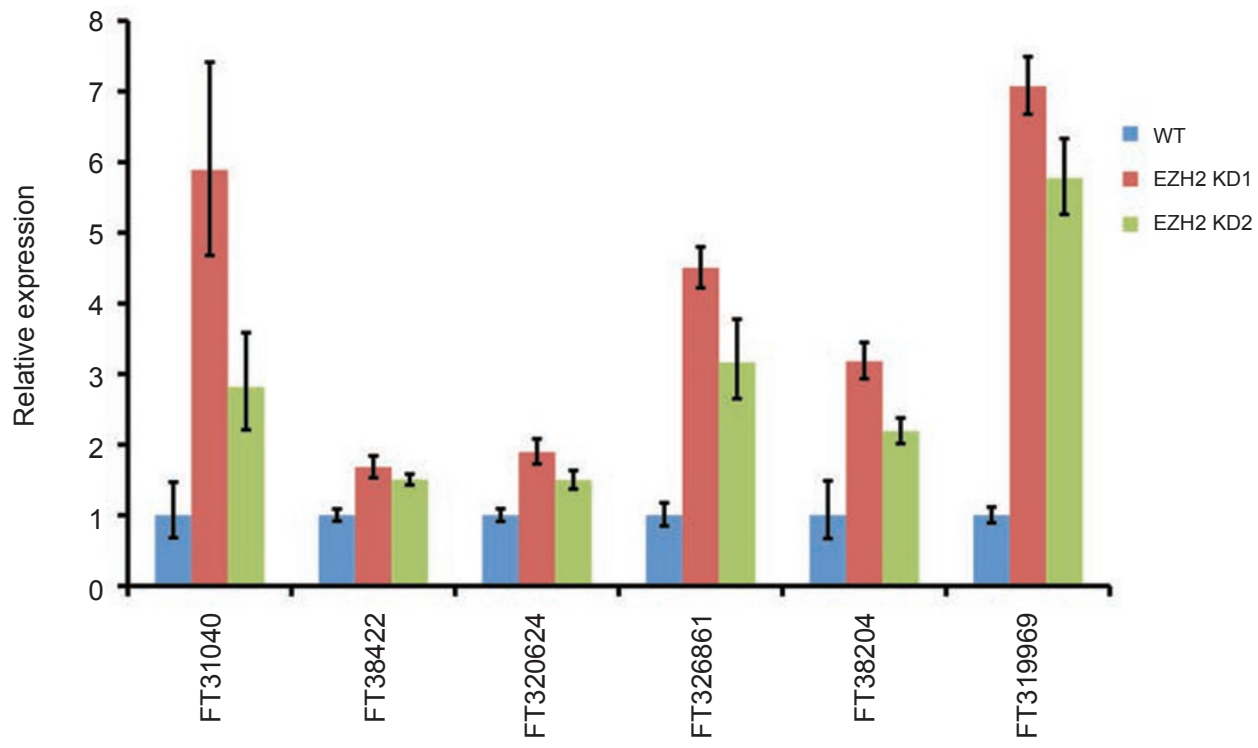

Figure 4 Ezh2-mediated H3K27 methylation represses IncRNA transcription in ES cells. (A) Relative mRNA levels of Ezh2 in wild-type and Ezh2 knockdown ES cells as determined by RT-qPCR. (B) Western blot analysis of H3K27me3 levels in Ezh2 KD ES cells. (C) Box plot presentation of expression changes (KD1/ES) in all IncRNAs $(n=24357)$ compared to IncRNAs marked by promoter H3K4me3 $(n=6$ 834) or H3K27me3 $(n=1$ 294) in ES cells. Significant $P$ values were determined using the Student's $t$ test. (D) Relative expression of IncRNAs upon Ezh2 knockdown in ES cells as determined by RT-qPCR. These IncRNAs were randomly selected from the list of IncRNAs that contain H3K27me3 in ES cells and are at least two-fold upregulated upon Ezh2 knockdown as determined by microarray analysis.

and H3K27 methylation of lncRNA promoters correlate with expression of IncRNAs. To this end, IncRNAs were categorized into two groups based on their expression levels in ES cells compared to both NPCs and fibroblasts (at least a two-fold change in ES versus NPCs/ fibroblasts). The average tag density for $\mathrm{H} 3 \mathrm{~K} 4 \mathrm{me} 3$ and
H3K27me3 was examined over a region of $+/-5 \mathrm{~kb}$ from the TSS using previously published data [25]. Similar to previous reports on protein-coding genes, levels of H3K4me3 peak at TSSs and are independent of expression status (Figure 3A). However, IncRNAs that were expressed higher in ES cells compared to NPCs/fibroblasts 
tended to have slightly higher levels of $\mathrm{H} 3 \mathrm{~K} 4 \mathrm{me} 3$. On the other hand, lncRNAs that were expressed at lower levels in ES cells exhibited a broad peak of H3K27me3 that sharply dropped after the TSS (Figure 3B). Levels of H3K27me3 remained consistently low in the same region when the opposite group was examined.

As mentioned above, previous studies of protein-coding genes in ES cells suggest that gene promoters marked by bivalent domains are thought to be poised for activation or repression upon differentiation [24]. To determine whether the same trend holds true for IncRNAs, we categorized lncRNAs that were bivalent in ES cells based on their modification state in fibroblast cells and examined their expression change upon differentiation. On average, bivalent lncRNAs that become $\mathrm{H} 3 \mathrm{~K} 4 \mathrm{me} 3$ in fibroblasts are expressed significantly higher in fibroblasts compared to ES cells, while those that become H3K27me3 are expressed significantly lower (Figure 3C). Collectively, the above results demonstrate that $\mathrm{H} 3 \mathrm{~K} 4 \mathrm{me} 3$ and $\mathrm{H} 3 \mathrm{~K} 27 \mathrm{me} 3$ can be correlated with the activation and repression of lncRNAs, respectively. Therefore, the relationship between transcription levels and promoter $\mathrm{H} 3 \mathrm{~K} 4 \mathrm{me} 3$ and $\mathrm{H} 3 \mathrm{~K} 27 \mathrm{me} 3$ levels previously discovered for protein-coding genes holds true for lncRNAs.

\section{Ezh2-mediated H3K27 methylation represses lncRNAs in ES cells}

We next asked whether the presence of H3K27me3 at the promoter affects the expression of IncRNAs in ES cells. To this end, we attempted to manipulate levels of H3K27me3 by knocking down Ezh2, the catalytic subunit of the Polycomb Repressive Complex 2 (PRC2) [26]. We generated lentiviruses expressing two independent shRNAs targeting Ezh2, which we used for transduction of ES cells. Following puromycin selection, knockdown efficiency was confirmed by RT-qPCR (Figure 4A). Consistent with down-regulation of Ezh2, global levels of H3K27me3 were dramatically reduced (Figure 4B).

We analyzed the lncRNA expression profile in Ezh2 KD ES cells using the custom-designed microarray described earlier and found that both knockdowns correlated well with each other $(\mathrm{Pcc}=0.935)$. To determine whether H3K27me3 plays a role in silencing lncRNAs in ES cells, we grouped lncRNAs based on the presence of $\mathrm{H} 3 \mathrm{~K} 4 \mathrm{me} 3$ or $\mathrm{H} 3 \mathrm{~K} 27 \mathrm{me} 3$ at their promoters and examined their expression change upon Ezh2 knockdown (Figure 4C). Not surprisingly, lncRNAs marked by H3K4me3 exhibited no significant changes in expression compared to all lncRNAs. On the other hand, we found that lncRNAs containing H3K27me3 tend to be expressed at a higher level upon Ezh2 knockdown when compared to those containing $\mathrm{H} 3 \mathrm{~K} 4 \mathrm{me} 3(P=1.415 \times$ $\left.10^{-10}\right)$ or all $\operatorname{lncRNAs}\left(P=9.64 \times 10^{-9}\right)$, indicating that Ezh2-mediated H3K27 methylation contributes to the repression of a subset of lncRNAs in ES cells.

To further analyze the effect of Ezh2 knockdown on lncRNA expression, we examined the microarray data and found $163 \operatorname{lncRNAs}$ that were at least two-fold upregulated in both knockdowns of Ezh2. Of these, 19 were also marked with H3K27me3 in ES cells. From this group, we randomly selected 6 lncRNAs and confirmed that their expression was de-repressed upon Ezh2 knockdown by RT-qPCR (Figure 4D). The effect of Ezh2 on these lncRNAs appears to be direct, as analysis of previously published genome-wide mapping data [27] indicated that the promoters of these IncRNAs are bound not only by Ezh2 but also by Suz12, another component of the PRC2 complex [26].

\section{Discussion}

In summary, our study examined the lncRNA expression profiles in ES, NPC, and fibroblasts and analyzed their relationship with their promoter $\mathrm{H} 3 \mathrm{~K} 4 \mathrm{me} 3$ and H3K27me3 levels. Our results indicate that, similar to protein-coding genes, H3K4me 3 and $\mathrm{H} 3 \mathrm{~K} 27 \mathrm{me} 3$ are generally correlated with IncRNA activation and repression, respectively. In addition, through shRNA-mediated knockdown of Ezh2 in ES cells, we demonstrate that Ezh2-mediated H3K27 methylation also functions to repress the expression of IncRNAs, thus extending the observations from protein-coding genes to lncRNAs.

Our finding that the Polycomb group of proteins regulates lncRNAs in addition to protein-coding genes prompted us to investigate whether transcription factors are also involved in regulating lncRNAs. Taking advantage of previously published genome-wide localization maps of transcription factors involved in ES cell biology [28], we examined the promoter regions of lncRNAs for the presence of transcription factors. Our analysis indicates that $30 \%$ (3 232 out of 10937 ) of the lncRNAs in our dataset contain at least one transcription factor bound to its promoter (Supplementary information, Figure S1A). Furthermore, the promoter regions of almost 300 lncRNAs contain at least three of the four core transcription factors important for ES cell maintenance (Nanog, Oct4, Sox2, and Klf4) (Supplementary information, Figure S1B). These results suggest that transcription factors may play a role in regulating lncRNAs. It is also possible that other regulatory mechanisms employed by protein coding genes may apply to lncRNAs.

Several lncRNAs including Xist, HOTAIR, and AIR have recently been demonstrated to be functional molecules important for specific biological processes [12, 13, 
15]. Given that over 30000 lncRNAs have been annotated in the FANTOM database, it is very likely that many more functional lncRNAs will be identified. In support of this prediction, we find that a subset of lncRNAs are differentially expressed and marked with different histone modifications, suggesting that these molecules play specific roles in different cell types. However, characterizing their biochemical and biological function is no trivial matter. Based on the few number of characterized lncRNAs, it is clear that lncRNAs can participate in various biological processes and function through diverse mechanisms $[9,14]$, thus making it difficult to predict their mechanism of action.

Despite these challenges, our demonstration that lncRNAs are regulated in a manner similar to that of proteincoding genes allows for identification of functional lncRNAs through a candidate-based approach by analyzing expression data as well as the presence or absence of specific histone modifications. Because we find that a subset of lncRNAs are developmentally regulated, it will be of great interest to determine if lncRNAs play a crucial role in ES cell biology. Our analysis indicates that there are 13 lncRNAs that contain H3K4me3 (and no H3K27me3) in ES cells in addition to $\mathrm{H} 3 \mathrm{~K} 27 \mathrm{me} 3$ (and no $\mathrm{H} 3 \mathrm{~K} 4 \mathrm{me} 3$ ) in either NPCs or fibroblasts. Of these, five lncRNAs are expressed at least two-fold higher in ES cells compared to NPCs or fibroblasts. These lncRNAs can be experimentally tested as candidates important for ES cell biology using a knockdown approach. A similar approach can be used to identify candidate lncRNAs that may be important for ES cell differentiation.

The production of diverse cell types in an organism has traditionally been attributed to differential gene expression that is controlled in part through epigenetic mechanisms such as histone modifications. However, emerging evidence demonstrating the differential expression of lncRNAs has indicated that lncRNAs may also contribute to this process. While many challenges remain with regard to determining the significance of these molecules, our work provides a resource for the scientific community to identify candidate lncRNAs that are important in ES cell biology. While only a fraction of annotated lncRNAs have currently been characterized, future work will undoubtedly uncover a larger biological role for lncRNAs than originally anticipated.

\section{Materials and Methods}

\section{IncRNA definitions and analysis}

The positions of 1 ncRNAs from the Fantom 3 database were extracted from http://jsm-research.imb.uq.edu.au/rnadb/ and used for all analyses in this work. Promoter regions were defined as 2 $\mathrm{kb}$ surrounding the start site (TSS) of each transcription unit as- sessed. Overlap analysis was carried out using previously defined datasets: annotated REFSEQ gene lists were procured as described in [29], H3K4me3 and H3K27me3 peaks observed in ES cells, NPCs, and mouse embryonic fibroblasts as described in [25], and genome-wide locations of transcription factors in ES cells as described in [28]. These analyses were carried out using the Python module FJOIN [30]. Whole-genome averaging of epigenetic mark enrichment was performed by normalizing $\mathrm{H} 3 \mathrm{~K} 4 \mathrm{me} 3$ and H3K27me3 ChIP-seq reads against whole-cell extract. The region from -5 to $+5 \mathrm{kB}$ relative to the TSS was divided into $200 \mathrm{bp}$ windows and the average tag density of the subdivided promoters was computed. Unless otherwise stated, only Fantom 3 entries that did not overlap with annotated REFSEQ genes or promoters were analyzed.

\section{Microarray analysis}

Total RNA was extracted from E14ps mES cells, mouse tail tip fibroblasts, and neurospheres, derived from E14ps mES cells using a previously published procedure [31]. RNA quality was assessed via Agilent Bioanlayzer. mRNA was amplified using the Low RNA Linear Amplification Kit (Agilent Technologies) by the standard manufacturer's protocol. Hybridization of experimental RNA labeled with Cy5 and Cy3-labeled Universal Reference RNA (Ambion) was carried out by using a standard protocol on a custom-designed array manufactured by Agilent Technologies. Briefly, three unique $60 \mathrm{nt}$ probes were designed for each entry in the Fantom3 database and were synthesized along with lineage marker control probes on a $2 \times 10^{5}-\mathrm{K}$ format array. Upon array scanning, data were quality filtered, normalized by the Lowess method, and all probes corresponding to a given lncRNA were averaged using the UNC Microarray Database (UNCMD). Intraclass correlation analysis was carried out in the UNCMD. Probe design files and microarray data have been submitted to the NCBI GEO database under accession number GSE22451.

\section{Ezh2 knockdown and RT-qPCR}

Hairpins targeting Ezh2 (KD1: 5'-GAA CTG AAA CCT TAA ACC A-3'; KD2: 5'-GTA TGT GGG CAT CGA ACG A-3') were cloned into a lentiviral vector under the control of the U6 promoter using previously published methods [32]. E14ps mES cells were infected with lentiviruses and subjected to puromycin selection. For RT-qPCR, $1 \mu \mathrm{g}$ of total RNA was subjected to reverse transcription using ImpromII kit (Promega). The resulting cDNA was diluted 1:5 and $5 \mu \mathrm{l}$ of the resulting solution was used for Real Time PCR analysis on an Applied Biosystems 7300 machine using SYBR GreenER (Invitrogen). Reactions were performed in triplicate. Gapdh was used as an endogenous control. Primer sequences can be found in Supplementary information, Table S2.

\section{Boxplots and statistics}

Boxplots were generated in the $\mathrm{R}$ package for defined subsets of genes. RT-qPCR statistics were computed using Student's $t$ test. Wilcoxon signed-rank test (Figure 3) and Student's $t$ test (Figure 4) were used to generate the presented $P$ values.

\section{Acknowledgments}

We thank Olena Taranova for providing the RNA and Jin He for generating the Ezh2 knockdown constructs. This work was 
supported by NIH (GM68804). Y.Z. is an Investigator of the Howard Hughes Medical Institute.

\section{References}

1 Birney E, Stamatoyannopoulos JA, Dutta A, et al. Identification and analysis of functional elements in $1 \%$ of the human genome by the ENCODE pilot project. Nature 2007; 447:799816.

2 Cheng J, Kapranov P, Drenkow J, et al. Transcriptional maps of 10 human chromosomes at 5-nucleotide resolution. Science 2005; 308:1149-1154.

3 Consortium IHGS. Finishing the euchromatic sequence of the human genome. Nature 2004; 431:931-945.

4 Johnson JM, Edwards S, Shoemaker D, Schadt EE. Dark matter in the genome: evidence of widespread transcription detected by microarray tiling experiments. Trends Genet 2005; 21:93-102.

5 Kapranov P, Cheng J, Dike S, et al. RNA maps reveal new RNA classes and a possible function for pervasive transcription. Science 2007; 316:1484-1488.

6 Dinger ME, Amaral PP, Mercer TR, et al. Long noncoding RNAs in mouse embryonic stem cell pluripotency and differentiation. Genome Res 2008; 18:1433-1445.

7 Mercer TR, Dinger ME, Sunkin SM, Mehler MF, Mattick JS. Specific expression of long noncoding RNAs in the mouse brain. Proc Natl Acad Sci USA 2008; 105:716-721.

8 Ravasi T, Suzuki H, Pang KC, et al. Experimental validation of the regulated expression of large numbers of non-coding RNAs from the mouse genome. Genome Res 2006; 16:11-19.

9 Ponting CP, Oliver PL, Reik W. Evolution and functions of long noncoding RNAs. Cell 2009; 136:629-641.

10 Guttman M, Amit I, Garber M, et al. Chromatin signature reveals over a thousand highly conserved large non-coding RNAs in mammals. Nature 2009; 458:223-227.

11 Ponjavic J, Ponting CP, Lunter G. Functionality or transcriptional noise? Evidence for selection within long noncoding RNAs. Genome Res 2007; 17:556-565.

12 Nagano T, Mitchell JA, Sanz LA, et al. The Air noncoding RNA epigenetically silences transcription by targeting G9a to chromatin. Science 2008; 322:1717-1720.

13 Rinn JL, Kertesz M, Wang JK, et al. Functional demarcation of active and silent chromatin domains in human HOX loci by noncoding RNAs. Cell 2007; 129:1311-1323.

14 Wilusz JE, Sunwoo H, Spector DL. Long noncoding RNAs: functional surprises from the RNA world. Genes Dev 2009; 23:1494-1504.

15 Zhao J, Sun BK, Erwin JA, Song JJ, Lee JT. Polycomb proteins targeted by a short repeat RNA to the mouse X chromosome. Science 2008; 322:750-756.
16 Cedar H, Bergman Y. Linking DNA methylation and histone modification: patterns and paradigms. Nat Rev Genet 2009; 10:295-304.

17 Martin C, Zhang Y. The diverse functions of histone lysine methylation. Nat Rev Mol Cell Biol 2005; 6:838-849.

18 Carninci P, Kasukawa T, Katayama S, et al. The transcriptional landscape of the mammalian genome. Science 2005; 309:1559-1563.

19 Carninci P, Sandelin A, Lenhard B, et al. Genome-wide analysis of mammalian promoter architecture and evolution. Nat Genet 2006; 38:626-635.

20 Ge X, Wu Q, Jung YC, Chen J, Wang SM. A large quantity of novel human antisense transcripts detected by LongSAGE. Bioinformatics 2006; 22:2475-2479.

21 Kapranov P, Willingham AT, Gingeras TR. Genome-wide transcription and the implications for genomic organization. Nat Rev Genet 2007; 8:413-423.

22 Katayama S, Tomaru Y, Kasukawa T, et al. Antisense transcription in the mammalian transcriptome. Science 2005; 309:1564-1566.

23 Schuettengruber B, Chourrout D, Vervoort M, Leblanc B, Cavalli G. Genome regulation by polycomb and trithorax proteins. Cell 2007; 128:735-745.

24 Bernstein BE, Mikkelsen TS, Xie X, et al. A bivalent chromatin structure marks key developmental genes in embryonic stem cells. Cell 2006; 125:315-326.

25 Mikkelsen TS, Ku M, Jaffe DB, et al. Genome-wide maps of chromatin state in pluripotent and lineage-committed cells. Nature 2007; 448:553-560.

26 Cao R, Wang L, Wang H, et al. Role of histone H3 lysine 27 methylation in Polycomb-group silencing. Science 2002; 298:1039-1043.

$27 \mathrm{Ku} \mathrm{M}$, Koche RP, Rheinbay E, et al. Genomewide analysis of PRC1 and PRC2 occupancy identifies two classes of bivalent domains. PLoS Genet 2008; 4:e1000242.

28 Chen $\mathrm{X}, \mathrm{Xu} \mathrm{H}$, Yuan $\mathrm{P}$, et al. Integration of external signaling pathways with the core transcriptional network in embryonic stem cells. Cell 2008; 133:1106-1117.

29 Kallin EM, Cao R, Jothi R, et al. Genome-wide uH2A localization analysis highlights Bmil-dependent deposition of the mark at repressed genes. PLoS Genet 2009; 5:e1000506.

30 Richardson JE. fjoin: simple and efficient computation of feature overlaps. J Comput Biol 2006; 13:1457-1464.

31 Ying QL, Smith AG. Defined conditions for neural commitment and differentiation. Methods Enzymol 2003; 365:327341 .

32 He J, Kallin EM, Tsukada Y, Zhang Y. The H3K36 demethylase $\mathrm{Jhdm} 1 \mathrm{~b} / \mathrm{Kdm} 2 \mathrm{~b}$ regulates cell proliferation and senescence through p15(Ink4b). Nat Struct Mol Biol 2008; 15:1169-1175.

(Supplementary information is linked to the online version of the paper on Cell Research website.) 